\title{
The apparent anticorrelation between the mass opacity of interstellar dust and the surface density of interstellar gas
}

\author{
F. D. Priestley ${ }^{\star}$ and A. P. Whitworth \\ School of Physics and Astronomy, Cardiff University, Queen's Buildings, The Parade, Cardiff CF24 3AA, UK
}

Accepted 2020 February 14. Received 2020 February 11; in original form 2019 December 9

\begin{abstract}
Recent analyses of Herschel observations suggest that in nearby disc galaxies the dust mass opacity at $500 \mu \mathrm{m}, \kappa_{500}$, decreases with increasing gas surface density, $\Sigma_{\text {ISM }}$. This apparent anticorrelation between $\kappa_{500}$ and $\Sigma_{\text {ISM }}$ is opposite to the behaviour expected from theoretical dust evolution models; in such models, dust in denser, cooler regions (i.e. regions of increased $\Sigma_{\text {ISM }}$ ) tends to grow and therefore to have increased $\kappa_{500}$. We show, using a toy model, that the presence of a range of dust temperatures along the line of sight can lead to spuriously low estimated values of $\kappa_{500}$. If in regions of higher $\Sigma_{\text {ISM }}$ the range of dust temperatures extends to lower values (as seems likely), the magnitude of this effect may be sufficient to explain the apparent anticorrelation between $\kappa_{500}$ and $\Sigma_{\text {ISM }}$. Therefore there may not be any need for spatial variation in the intrinsic dust properties that run counter to theoretical expectations.
\end{abstract}

Key words: opacity-dust, extinction-galaxies: ISM.

\section{INTRODUCTION}

Dust is an important constituent of the interstellar medium (ISM), making up $\sim 1$ per cent of the total mass, and locking up around half of the available metals (Draine 2011). Far-infrared (IR) observations of thermal dust emission are an important tool in investigating the properties of both the dust and the ISM at large (Kennicutt et al. 2009; Eales et al. 2012). However, converting observed fluxes into physical quantities requires knowledge of the optical properties of interstellar dust at far-IR and submillimetre wavelengths. A commonly used simplification is to fit the spectral energy distribution (SED) with a modified blackbody (MBB) function,

$S_{\lambda}=\frac{M_{\mathrm{d}} \kappa_{\lambda} B_{\lambda}(T)}{D^{2}}$,

where $S_{\lambda}$ is the observed flux density, $M_{\mathrm{d}}$ is the dust mass, $\kappa_{\lambda}$ is the dust mass opacity (i.e. the opacity per unit mass of dust) at wavelength $\lambda, B_{\lambda}(T)$ is the Planck function (i.e. the blackbody intensity), $T$ is the dust temperature, and $D$ is the source distance. Equation (1) is only accurate if all the dust on a given line of sight (a) is in thermal equilibrium, (b) has the same temperature, and (c) is optically thin to its own emission (generally true in the far-IR). $\kappa_{\lambda}$ can be measured in the laboratory, or calculated (if the dust composition and the distributions of size and shape are specified). The variation of $\kappa_{\lambda}$ with wavelength can usually be approximated with a power law in the far-IR and submillimetre (e.g. fig. 4 from

^E-mail: priestleyf@ cardiff.ac.uk
Galliano, Galametz \& Jones 2018), viz.

$\kappa_{\lambda} \simeq \kappa_{0}\left(\frac{\lambda_{0}}{\lambda}\right)^{\beta}$

here $\kappa_{0}$ is the mass opacity at the reference wavelength $\lambda_{0}$. If $\kappa_{0}$ and $D$ are known, $M_{\mathrm{d}}, T$, and $\beta$ can - in principle - be fit as free parameters.

The value of $\kappa_{0}$ used can be based on laboratory measurements (e.g. Jaeger et al. 1994; Zubko et al. 1996) or on theoretical calculations for a particular material (e.g. Laor \& Draine 1993), but usually an average value is adopted, accounting for a range of dust compositions and sizes, constrained by observational features such as the extinction curve and elemental depletions, and in some cases also taking account of the evolutionary processes undergone by dust in different phases of the ISM (e.g. Draine \& Li 2007; Jones et al. 2016). Derived dust properties are therefore very model dependent, and although most commonly used values for $\kappa_{0}$ agree within a factor of order 6, the full range is at least a factor of 300 (see table D1 in Whitworth et al. 2019). Moreover, there is no guarantee that any of the models invoked accurately reproduce the true properties of interstellar dust, or that the same model is appropriate in all environments (i.e. different phases of the ISM, galaxies with different stellar and/or gas properties).

James et al. (2002) have proposed a way to estimate $\kappa_{0}$ in galaxies directly, by determining the mass and metallicity of the interstellar gas, and assuming a constant fraction of the metallicity is locked up in dust; this gives a value for the dust mass independent of the far-IR data. The fraction of the metallicity locked up in dust grains $(0.5 \pm 0.1)$ appears to be relatively constant in the local Universe (e.g. Jenkins 2009; Peeples et al. 2014), and so 
the errors introduced by this assumption are probably lower than those affecting theoretical estimates for $\kappa_{0}$. The measured value of $\kappa_{0}$ can then be used to analyse dust in other galaxies, and to calibrate theoretical dust models. Clark et al. (2016) present an updated estimate of $\kappa_{0}$ obtained by this procedure, taking advantage of improved far-IR observations from Herschel. They find $\kappa_{500} \sim 0.5 \mathrm{~cm}^{2} \mathrm{~g}^{-1}$ (where $\kappa_{500}$ is $\kappa_{\lambda}$ at $\lambda=500 \mu \mathrm{m}$ ). This is somewhat smaller than the values predicted by recent theoretical models (e.g. Jones et al. 2016), but consistent with some earlier models (e.g. Draine 2003).

Recently, Clark et al. (2019) have applied this method to spatially resolved observations of the two galaxies M74 (NGC 628) and M83 (NGC 5236); they conclude that $\kappa_{500}$ tends to decrease with increasing ISM surface density, $\Sigma_{\text {ISM }}$. This is in contrast with what is expected from dust evolution models, where grains in denser regions of the ISM are expected to be larger and therefore to have higher far-IR opacities (e.g. Köhler, Ysard \& Jones 2015; Ysard et al. 2018). Clark et al. (2019) find this apparent anticorrelation between $\kappa_{500}$ and $\Sigma_{\text {ISM }}$ to be robust against systematic effects that might have affected their methodology. Bianchi et al. (2019) analysed a large sample of galaxies and found that the dust emissivity at $250 \mu \mathrm{m}$ appears to decrease with increasing molecular gas fraction. If interpreted as a decline in the opacity, this would appear to support the $\kappa_{500}-\Sigma_{\text {ISM }}$ anticorrelation. However, the calculation of $\kappa_{500}$ depends on the single dust temperature returned by a singletemperature $\mathrm{MBB}$ fit (hereafter, a $1 \mathrm{MBB}$ fit). If there is a range of temperatures on the line of sight, this estimate, being flux-weighted, is biased towards the higher temperatures. The procedure used by Clark et al. (2019) then tends to underestimate $\kappa_{500}$ in order to compensate for this temperature bias. In this Letter, we show that, with reasonable physical assumptions, this temperature bias can produce an apparent anticorrelation between $\kappa_{500}$ and $\Sigma_{\text {ISM }}$, similar in magnitude to that found by Clark et al. (2019), without any underlying variation in the intrinsic optical properties of the dust.

\section{METHOD}

Clark et al. (2019) estimate $\kappa_{500}$ by rearranging equation (1) so that

$\kappa_{500}=\frac{D^{2} S_{500}}{M_{\mathrm{d}} B_{500}(T)}$,

with $M_{\mathrm{d}}$ calculated from the gas mass, the metallicity, and the assumed fraction of the metallicity locked up in dust grains. The dust temperature is estimated by fitting a single-temperature MBB to the Herschel far-IR fluxes. As the emitted flux increases with temperature at all wavelengths, a given mass of warmer dust will contribute more to the total emission than the same mass of cooler dust, particularly at shorter wavelengths, thereby biasing the values returned by the MBB fit (Shetty et al. 2009). As a simple illustration of this effect, Fig. 1 shows the monochromatic fluxes from two MBBs, calculated using equations (1) and (2), and the sum of these fluxes. We choose typical (but arbitrary) values of $M_{\mathrm{d}}=10^{5} \mathrm{M}_{\odot}$, $D=1 \mathrm{Mpc}, \beta=2$, and $\kappa_{500}=2 \mathrm{~cm}^{2} \mathrm{~g}^{-1}$. Fitting a single MBB to the sum of the fluxes gives a temperature of $21 \mathrm{~K}$, and a slightly reduced $\beta=1.8$. Substituting the total mass of $2 \times 10^{5} \mathrm{M}_{\odot}$ into equation (3) then returns a value of $\kappa_{500}=1.1 \mathrm{~cm}^{2} \mathrm{~g}^{-1}$, nearly a factor of 2 smaller than the true value.

Clark et al. (2019) acknowledge this issue, but argue that this will only systematically lower their measured $\kappa_{500}$ from the true value. They also note that repeating their analysis using a twotemperature $\mathrm{MBB}$ fit (hereafter, a $2 \mathrm{MBB}$ fit) does not remove the anticorrelation between $\kappa_{500}$ and $\Sigma_{\text {ISM }}$. However, they do not

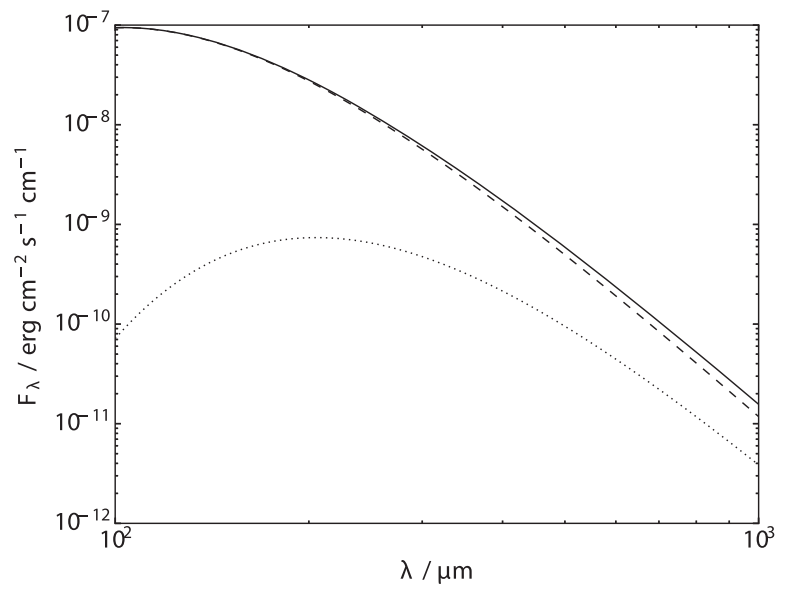

Figure 1. The fluxes from MBBs with identical mass of $T=20 \mathrm{~K}$ (dashed line) and $T=10 \mathrm{~K}$ (dotted line), and their sum (solid line). Even at long wavelengths the $10 \mathrm{~K}$ contribution is small.

appear to have considered the possibility that the temperature bias might be correlated with the ISM surface density, $\Sigma_{\text {ISM }}$, in the sense that at larger column density, attenuation of the radiation field by dust extinction is likely to be greater, leading to both lower dust temperatures and/or a higher proportion of dust at lower temperatures.

We investigate this effect by using a toy model to generate synthetic dust SEDs, and then using the same procedure as Clark et al. (2019) to estimate $\kappa_{500}$ from these synthetic dust SEDs. The toy model has two parameters, $f_{\text {att }}$ and $\Sigma_{\text {ISM }}$. A fraction $\left(1-f_{\text {att }}\right)$ of the modelled dust is heated by an unattenuated radiation field, and the remainder, $f_{\text {att }}$, by an attenuated radiation field. The attenuation is related to $\Sigma_{\text {ISM }}$ using the standard ratio between visual extinction, $A_{\mathrm{V}}$, and the column density of hydrogen in all forms, $N_{\mathrm{H}}$ (Bohlin, Savage \& Drake 1978), viz.

$A_{\mathrm{V}} \simeq 0.6 \mathrm{mag}\left(\frac{N_{\mathrm{H}}}{10^{21} \mathrm{Hcm}^{-2}}\right) \simeq 0.052 \mathrm{mag}\left(\frac{\Sigma_{\mathrm{ISM}}}{\mathrm{M}_{\odot} \mathrm{pc}^{-2}}\right)$.

Although $f_{\text {att }}$ is probably correlated with $\Sigma_{\text {ISM }}$, we treat $f_{\text {att }}$ and $\Sigma_{\text {ISM }}$ as independent free parameters, so as to avoid additional assumptions.

The synthetic SEDs are generated using the dust heating code DINAMO (Priestley, Barlow \& De Looze 2019), which accounts for stochastic heating of small grains. We assume that dust is heated by the Mathis, Mezger \& Panagia (2009) interstellar radiation field, and attenuation is implemented using the Cardelli, Clayton \& Mathis (1989) extinction law. We adopt a power-law distribution of grain sizes between 0.005 and $0.25 \mu \mathrm{m}$, with exponent -3.5 (Mathis, Rumpl \& Nordsieck 1977). We compute the dust properties using optical constants for amorphous carbon from Zubko et al. (1996). We address the possible evolution of the dust properties with $\Sigma_{\text {ISM }}$ in Appendix A. We emphasize that, since the temperature bias is a differential effect, the results we present below are not sensitive to these choices of radiation field, grain-size distribution, or grain material.

We convert the dust SED into photometric fluxes for the Photodetector Array Camera and Spectrometer (PACS) and Spectral and Photometric Imaging Receiver (SPIRE) bands using the appropriate filter response curves, and assume error bars of 10 per cent for each band, typical of the average values of the DustPedia sample (Davies et al. 2017) from which Clark et al. (2019) take their far-IR data. We can then derive the value of $\kappa_{500}$ that would be inferred 


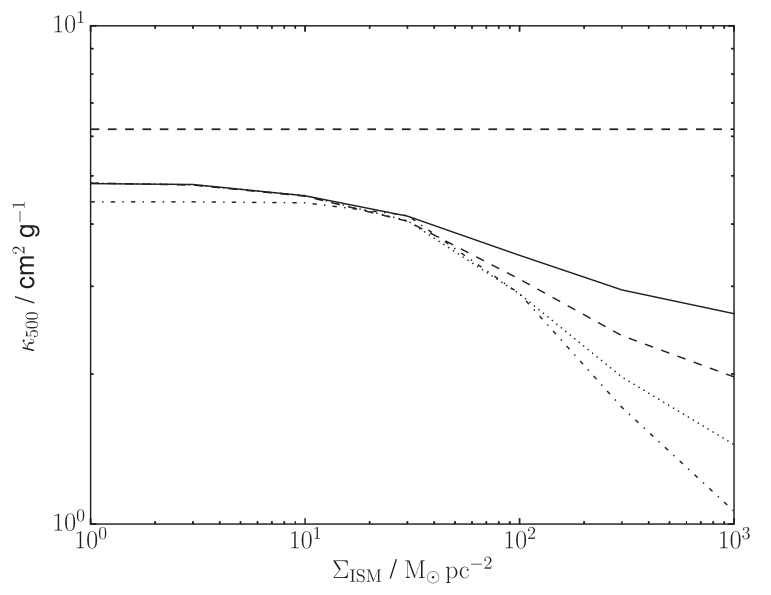

Figure 2. The inferred value of $\kappa_{500}$ as a function of $A_{\mathrm{V}}$, obtained using a single-temperature $\mathrm{MBB}$ fit (1MBB fit). The extinction, $A_{\mathrm{V}}$, which determines the attenuation of the radiation field, is related to the surface density, $\Sigma_{\text {ISM }}$, by equation (4). The different curves represent cases where different fractions of dust, $f_{\text {att }}$, are located in regions where the radiation field is attenuated: $f_{\text {att }}=0.5$ (solid line), 0.667 (dashed line), 0.75 (dotted line), and 0.9 (dot-dashed line). The thin dashed line shows the true value of $\kappa_{500}=6.2 \mathrm{~cm}^{2} \mathrm{~g}^{-1}$.

using the Clark et al. (2019) procedure, as a function of $\Sigma_{\text {ISM }}$, for representative values of $f_{\text {att }}$. For the purpose of illustration, we again assume a source distance of $D=1 \mathrm{Mpc}$ and a total dust mass $M_{\mathrm{d}}=10^{5} \mathrm{M}_{\odot}$. However, since the dependence on these parameters is limited to $S_{\lambda} \propto M_{\mathrm{d}} / D^{2}$ (see equation 3), this does not affect the calculation of $\kappa_{500}$.

We note that there are two distinct effects producing a range of temperatures. First, even if the radiation field heating the dust were the same everywhere, dust grains of different composition and/or different size would have different temperatures; the smallest dust grains would also have time-varying temperatures. Second, the radiation field heating the dust is not the same everywhere (this is what we parametrize, very simplistically, with $f_{\text {att }}$ ), and therefore even large dust grains of the same composition and size have different temperatures in different locations.

\section{RESULTS}

Fig. 2 shows how the estimated $\kappa_{500}$ varies with $\Sigma_{\text {ISM }}$, for different discrete values of $f_{\text {att }}$ between 0.5 and 0.9 . For clarity, we do not show error bars, but uncertainties are 10 per cent or lower, for all combinations of $f_{\text {att }}$ and $\Sigma_{\text {ISM }}$. The inferred $\kappa_{500}$ values are always below the true value $\left(6.2 \mathrm{~cm}^{2} \mathrm{~g}^{-1}\right)$, even when there is no attenuation, because a $1 \mathrm{MBB}$ fit cannot account for multiple grain sizes, each with their own temperature (or temperatures in the case of very small stochastically heated grains). Using a $1 \mathrm{MBB}$ fit (Fig. 2), the derived $\kappa_{500}$ decreases monotonically with $\Sigma_{\text {ISM }}$ for all values of $f_{\text {att }}$, and by a factor of between 2 and 5 over two orders of magnitude in $\Sigma_{\text {ISM }}$, depending on the value of $f_{\text {att }}$. Qualitatively this reproduces the trend seen by Clark et al. (2019), without any change in the underlying dust optical properties.

The decrease is entirely due to fitting with single-temperature MBBs, SEDs that have been generated from a wide range of dust temperatures. This is shown in Fig. 3, where the bias towards the temperature of the dust heated by the unattenuated flux is apparent. Temperature values from the $1 \mathrm{MBB}$ fits range between 18 and $22 \mathrm{~K}$, and all fits return $\beta \simeq 1.0$. The dust opacity we use actually has

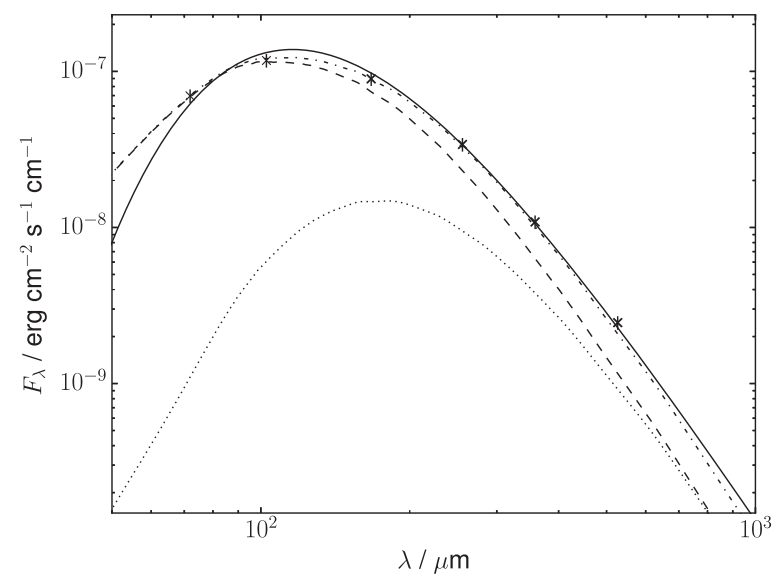

Figure 3. Fluxes of the unattenuated (dashed line) and attenuated (dotted line) dust components for $f_{\text {att }}=0.667$ and $\Sigma_{\mathrm{ISM}}=100 \mathrm{M}_{\odot} \mathrm{pc}^{-2}$ (hence $A_{\mathrm{V}}=5.2 \mathrm{mag}$ ), their combined flux (dot-dashed line) and the best-fitting MBB with $T=20.7 \mathrm{~K}$ and $\beta=1.0$ (solid line). The photometric fluxes and error bars are marked as crosses.

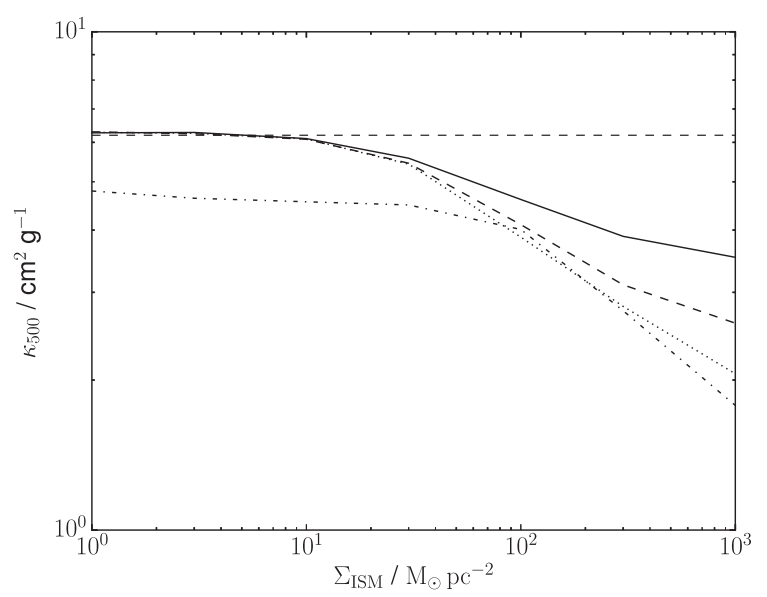

Figure 4. As Fig. 2, but for a radiation field strength increased by a factor of 10 .

$\beta \simeq 1.4$, but fixing $\beta$ to this value does not significantly change the results; the $\kappa_{500}$ values returned by the $1 \mathrm{MBB}$ fits are slightly higher, but they still decrease with increasing $\Sigma_{\text {ISM }}$, and by essentially the same factor over the same range of $\Sigma_{\text {ISM }}$. Increasing the radiation field strength by a factor of 10 also fails to eliminate this trend, although in this case, shown in Fig. 4 , the inferred values of $\kappa_{500}$ at lower $\Sigma_{\text {ISM }}$ and lower $f_{\text {att }}$ are in better agreement with the true value.

Quantitatively, the opacities estimated by Clark et al. (2019) decrease with increasing surface density, $\Sigma_{\text {ISM }}$, somewhat faster and at somewhat lower values of $\Sigma_{\text {ISM }}$ than in our toy model. Specifically, for $\Sigma_{\text {ISM }}$ between 10 and $100 \mathrm{M}_{\odot} \mathrm{pc}^{-2}$, where the majority of the data in Clark et al. (2019) lie, we find a powerlaw exponent, $\mathrm{d} \ln \left(\kappa_{500}\right) / \mathrm{d} \ln \left(\Sigma_{\mathrm{ISM}}\right)$, between -0.14 and -0.28 , whereas Clark et al. (2019) find $\mathrm{d} \ln \left(\kappa_{500}\right) / \mathrm{d} \ln \left(\Sigma_{\text {ISM }}\right) \sim-0.35$ for both the galaxies they analyse (M74 and M83). However, there are two factors that in a more sophisticated model would reduce this discrepancy, by making the model slope steeper (i.e. more negative). First, $f_{\text {att }}$ is very likely correlated with $\Sigma_{\text {ISM }}$. Second, the ISM has substructure on scales below those resolved by the observations $(\sim 100 \mathrm{pc})$; consequently the radiation field reaching the dust in 


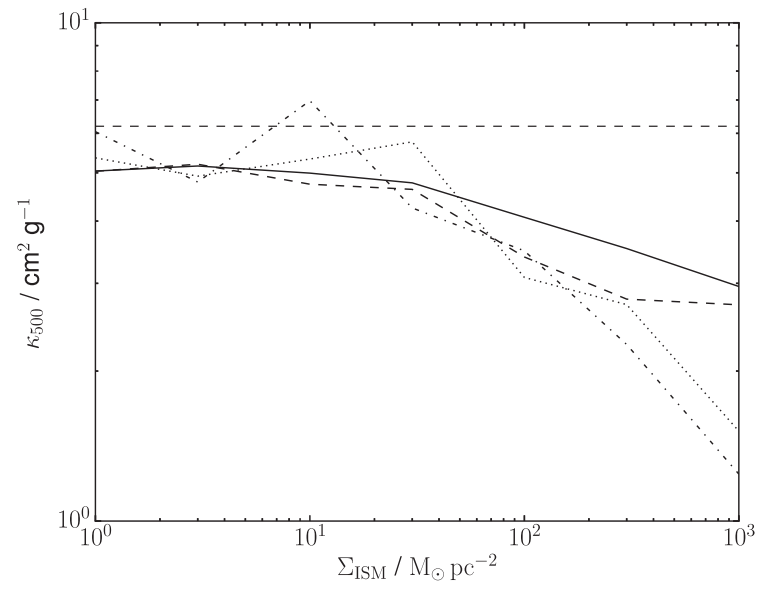

Figure 5. As Fig. 2, but for a two-temperature MBB fit (2MBB fit).

these unresolved substructures is more strongly attenuated than the model predicts, and the dust is cooler. If the model were able to take these two factors into account, the slope predicted by the model would be steeper, and the decrease in $\kappa_{500}$ would occur at lower values of $\Sigma_{\text {ISM. }}$. Given the significant error bars on the observed values, and the simplicity of our model, we conclude that the apparent anticorrelation between $\kappa_{500}$ and $\Sigma_{\text {ISM }}$ might be due partly, or even entirely, to the temperature bias being stronger in regions of high $\Sigma_{\text {ISM }}$.

Clark et al. (2019) find that, if they repeat their analysis using a $2 \mathrm{MBB}$ fit, there is still an apparent anticorrelation between the inferred $\kappa_{500}$ and $\Sigma_{\text {ISM }}$, and we find the same. Fig. 5 shows the results we obtain with a two-temperature fit. The second MBB results in a slightly higher inferred $\kappa_{500}$, closer to the true value, but the anticorrelation with $\Sigma_{\text {ISM }}$ remains. The uncertainties in $\kappa_{500}$ are also much larger than for the single-temperature fit ( $\sim 40$ per cent). Although in principle the $2 \mathrm{MBB}$ fit might be expected to account for the colder dust missed by the $1 \mathrm{MBB}$ fit, there is no guarantee that a $2 \mathrm{MBB}$ fit will return mass fractions and temperatures even approximately representative of the true values. In addition (and as also noted by Clark et al. 2019), using two MBBs with six free parameters overfits the data when only five points (two PACS and three SPIRE bands) are available; this is the cause of the nonmonotonic trends in Fig. 5.

\section{DISCUSSION}

We have shown that a relatively simple physical model can produce a spurious apparent anticorrelation between $\kappa_{500}$ and $\Sigma_{\text {ISM }}$, similar in magnitude to the one reported by Clark et al. (2019), when analysed with the same procedure they use. The critical feature of the model is that on any line of sight there is a range of dust temperatures, and this range extends to lower temperatures on lines of sight with higher surface density (and hence, implicitly, higher attenuation of the radiation field heating some of the dust). Whether this is the correct explanation for the apparent anticorrelation, rather than a spatial variation in the intrinsic optical properties of the dust, or some combination of the two, is unclear. The simplistic model explored here assumes that, on any line of sight, a fraction $\left(1-f_{\text {att }}\right)$ of the dust grains is exposed to an unattenuated radiation field, and a fraction $f_{\text {att }}$ is exposed to a radiation field attenuated by an $A_{\mathrm{V}}$ that is proportional to the surface density of the ISM, $\Sigma_{\text {ISM }}$, on that line of sight (see equation 4$)$. There are two effects that the simple model does not include, but which would probably improve correspondence between the model predictions and the observations.

First, it seems likely that the fraction of dust heated by an attenuated radiation field, $f_{\text {att }}$, increases with the surface density of the interstellar gas, $\Sigma_{\text {ISM }}$; in other words $f_{\text {att }}$ is positively correlated with $\Sigma_{\text {ISM }}$. This would generate a single plot of $\kappa_{500}$ against $\Sigma_{\text {ISM }}$ in Figs 2 and 5, with a steeper (negative) slope. Provided it did not produce too large an effect, this would bring the slope of the plot closer to that determined by Clark et al. (2019).

Second, the pixel size used by Clark et al. (2019) corresponds to a few $100 \mathrm{pc}$ at the distances of the galaxies studied. On these scales, we expect each pixel to contain significant unresolved substructure (e.g. Elmegreen 2002). If a significant fraction of the dust is contained in unresolved substructures with low volumefilling factor but high density, this dust experiences a much more strongly attenuated radiation field than our toy model delivers (the model only invokes an attenuation corresponding to the surface density averaged over a pixel). Consequently the coefficient on the right-hand side of equation (4) should be increased, and the distribution of dust temperatures should extend to even lower values, thereby increasing the temperature bias. This will shift the curves in Figs 2, 4, and 5 to smaller values of $\Sigma_{\text {ISM. }}$. Again, provided it did not produce too large an effect, this would bring the plot closer to that observed by Clark et al. (2019).

Although we adopt the Mathis et al. (2009) radiation field, which is designed to reproduce conditions in the solar vicinity, the effect we have modelled is a differential one, and therefore we can expect similar results in regions where the radiation field is different, as indicated by the similar trends obtained with an enhanced radiation field (see Fig. 4). A more compelling evaluation of the effect we have considered would require more detailed and realistic modelling, including variations in the ambient unattenuated radiation field and $3 \mathrm{D}$ radiative transfer for a distribution of stellar radiation sources and dust (e.g. De Looze et al. 2014; Draine et al. 2014; Williams et al. 2019). We have also used constant dust optical properties, whereas in reality these would be expected to vary with $\Sigma_{\text {ISM }}$. In Appendix A, we demonstrate that the effect on the inferred value of $\kappa_{500}$ is strongly dependent on the assumed variation. Therefore, it is not possible to draw any robust conclusions about the behaviour of the dust opacity using the model developed here. However, it is clear from the results we report here that temperature bias must play a critical role in the interpretation of far-IR and submillimetre dust continuum observations like those from Herschel. The apparent anticorrelation between $\kappa_{500}$ and $\Sigma_{\text {ISM }}$ may be an entirely spurious consequence of this bias.

\section{ACKNOWLEDGEMENTS}

FDP and APW gratefully acknowledge the support of a Consolidated Grant (ST/K00926/1) from the UK Science and Technology Facilities Council (STFC). We also thank the referee, Simone Bianchi, for his comments, which helped us to improve the original version of this Letter.

\section{REFERENCES}

Bianchi S. et al., 2019, A\&A, 631, A102

Bohlin R. C., Savage B. D., Drake J. F., 1978, ApJ, 224, 132

Cardelli J. A., Clayton G. C., Mathis J. S., 1989, ApJ, 345, 245

Clark C. J. R., Schofield S. P., Gomez H. L., Davies J. I., 2016, MNRAS, 459,1646 
Clark C. J. R. et al., 2019, MNRAS, 489, 5256

Davies J. I. et al., 2017, PASP, 129, 044102

De Looze I. et al., 2014, A\&A, 571, A69

Draine B. T., 2003, ARA\&A, 41, 241

Draine B. T., 2011, Physics of the Interstellar and Intergalactic Medium. Princeton Univ. Press, Princeton, NJ

Draine B. T., Li A., 2007, ApJ, 657, 810

Draine B. T. et al., 2014, ApJ, 780, 172

Eales S. et al., 2012, ApJ, 761, 168

Elmegreen B. G., 2002, ApJ, 564, 773

Galliano F., Galametz M., Jones A. P., 2018, ARA\&A, 56, 673

Jaeger C., Mutschke H., Begemann B., Dorschner J., Henning T., 1994, A\&A, 292, 641

James A., Dunne L., Eales S., Edmunds M. G., 2002, MNRAS, 335 , 753

Jenkins E. B., 2009, ApJ, 700, 1299

Jones A. P., Köhler M., Ysard N., Dartois E., Godard M., Gavilan L., 2016, A\&A, 588, A43

Kennicutt R. C., Jr et al., 2009, ApJ, 703, 1672

Köhler M., Ysard N., Jones A. P., 2015, A\&A, 579, A15

Laor A., Draine B. T., 1993, ApJ, 402, 441

Mathis J. S., Rumpl W., Nordsieck K. H., 1977, ApJ, 217, 425

Mathis J. S., Mezger P. G., Panagia N., 2009, A\&A, 500, 259, Special Issue 500/01: reprint of 1983, A\&A, 128, 212

Peeples M. S., Werk J. K., Tumlinson J., Oppenheimer B. D., Prochaska J. X., Katz N., Weinberg D. H., 2014, ApJ, 786, 54

Priestley F. D., Barlow M. J., De Looze I., 2019, MNRAS, 485, 440

Shetty R., Kauffmann J., Schnee S., Goodman A. A., Ercolano B., 2009, ApJ, 696, 2234

Whitworth A. P. et al., 2019, MNRAS, 489, 5436

Williams T. G., Baes M., De Looze I., Relaño M., Smith M. W. L., Verstocken S., Viaene S., 2019, MNRAS, 487, 2753

Ysard N., Jones A. P., Demyk K., Boutéraon T., Koehler M., 2018, A\&A, $617, \mathrm{~A} 124$
Zubko V. G., Mennella V., Colangeli L., Bussoletti E., 1996, MNRAS, 282, 1321

\section{APPENDIX A: NON-CONSTANT DUST OPTICAL PROPERTIES}

While we have shown that an apparent anticorrelation between $\kappa_{500}$ and $\Sigma_{\text {ISM }}$ can be produced by a constant dust opacity, the trend predicted by dust evolution models is for $\kappa_{500}$ to increase with increasing $\Sigma_{\text {ISM }}$. The exact observed trend produced by a given dust model will depend not only on the optical constants, but also the relation between $\Sigma_{\text {ISM }}$ and the volume density of the gas, which is the relevant quantity for dust evolution (among others). This is beyond the scope of this Letter, but we can investigate the likely effect by increasing the value of $\kappa_{500}$ for the attenuated dust component, to represent grain growth in the denser gas.

We do this by changing the minimum and maximum sizes of our power-law grain size distribution to 10 and $20 \mu \mathrm{m}$, respectively, which increases the opacity to $\kappa_{500}=16.8 \mathrm{~cm}^{2} \mathrm{~g}^{-1}$. This results in a flat relation between the inferred $\kappa_{500}$ and $\Sigma_{\text {ISM }}$ for a given $f_{\text {att }}$, since the temperature of the attenuated grains is lower, and so the flux is entirely dominated by the unattenuated component. A more moderate increase in the grain size distribution to between 0.1 and $1 \mu \mathrm{m}$ for the attenuated component results in a much smaller increase in $\kappa_{500}$, and a negligible change to the results in Fig. 2. Whether the results of Clark et al. (2019) are consistent with an increasing dust opacity with gas density therefore depends on the details of the dust evolution model under consideration.

This paper has been typeset from a $\mathrm{T}_{\mathrm{E}} \mathrm{X} / \mathrm{L} \mathrm{T} \mathrm{E}$ file prepared by the author. 